\title{
Host-mediated post-translational prenylation of novel Dot/ Icm-translocated effectors of Legionella pneumophila
}

\author{
ChristopherT. D. Price ${ }^{1}$, Snake C. Jones ${ }^{1}$, Karen E. Amundson ${ }^{2}$ and Yousef Abu Kwaik 1,2,3,4* \\ Department of Microbiology and Immunology, College of Medicine, University of Louisville, Louisville, KY, USA \\ 2 Department of Biology, University of Louisville, Louisville, KY, USA \\ ${ }^{3}$ Department of Microbiology, Al-Quds University Medical School, Abu Dies, Jerusalem, Palestinian Territory \\ ${ }^{4}$ Department of Microbiology and Molecular Genetics, The Hebrew University Medical School, Jerusalem, Israel
}

\section{Edited by:}

Amal Amer, The Ohio State University USA

\section{Reviewed by:}

Amal Amer, The Ohio State University, USA

Estelle Cormet-Boyaka, The Ohio State University, USA

\section{*Correspondence:}

Yousef Abu Kwaik, School of Medicine, University of Louisville, 412 Building A, Louisville, KY 40202, USA

e-mail:abukwaik@louisville.edu
The Dot/lcm type IV translocated Ankyrin B (AnkB) effector of Legionella pneumophila is modified by the host prenylation machinery that anchors it into the outer leaflet of the Legionellacontaining vacuole (LCV), which is essential for biological function of the effector in vitro and in vivo. Prenylation involves the covalent linkage of an isoprenoid lipid moiety to a C-terminal CaaX motif in eukaryotic proteins enabling their anchoring into membranes. We show here that the LCV harboring an ankB null mutant is decorated with prenylated proteins in a Dot/lcmdependent manner, indicating that other LCV membrane-anchored proteins are prenylated. In silico analyses of four sequenced $L$. pneumophila genomes revealed the presence of eleven other genes that encode proteins with a C-terminal eukaryotic CaaX prenylation motif. Of these eleven designated Prenylated effectors of Legionella (Pel), seven are also found in L. pneumophila AA100. We show that six L. pneumophila AA100 Pel proteins exhibit distinct cellular localization when ectopically expressed in mammalian cells and this is dependent on action of the host prenylation machinery and the conserved cysteine residue of the CaaX motif. Although inhibition of the host prenylation machinery completely blocks intra-vacuolar proliferation of $L$. pneumophila, it only had a modest effect on intracellular trafficking of the LCV. Five of the Pel proteins are injected into human macrophages by the Dot/lcm type IV translocation system of $L$. pneumophila. Taken together, the Pel proteins are novel Dot/lcm-translocated effectors of L. pneumophila that are post-translationally modified by the host prenylation machinery, which enables their anchoring into cellular membranes, and the prenylated effectors contribute to evasion of lysosomal fusion by the LCV.

Keywords: Legionnaires', Agrobacterium, CaaX, Dot/lcm, prenylation, ankyrin, AnkB, Pel, F-box,RCE-1, IcmT, PFT, PGGT

\section{INTRODUCTION}

Exploitation of eukaryotic cellular processes is essential for proliferation of intracellular microbial pathogens. The Legionnaires' disease causing bacterium, Legionella pneumophila, replicates within alveolar macrophages causing pneumonia (Isberg et al., 2009). The organism is transmitted to humans from the aquatic environment where L. pneumophila replicates within ameba and ciliates (Molmeret et al., 2005; Franco et al., 2009). Co-evolution and adaptation of $L$. pneumophila to the intracellular lifestyle within ameba in the aquatic environment is believed to have played a major role in its ability to exploit evolutionarily conserved eukaryotic processes that enables its proliferation within human alveolar macrophages (Molmeret et al., 2005; Franco et al., 2009). Within both evolutionarily distant host cells, $L$. pneumophila evades endocytic fusion and intercepts ER-to-Golgi vesicle traffic to remodel its phagosome into an ER-derived vacuole (Kagan and Roy, 2002; Molmeret et al., 2005; Shin and Roy, 2008; Isberg et al., 2009).

The L. pneumophila Dot/Icm type IV secretion system (Segal et al., 1998; Vogel et al., 1998) injects into the host cell a cadre of $\sim 200$ effectors to modulate a myriad of cellular processes to re-program the host cell into a proliferation niche (de Felipe et al., 2008; Shin and Roy, 2008; Isberg et al., 2009). The Ankyrin B (AnkB) effector is injected into the host cell by the Dot/Icm system upon bacterial attachment to the plasma membrane, and exploits an evolutionarily conserved eukaryotic machinery within mammalian and protozoan cells (Al-Khodor et al., 2008, 2010a,b; Habyarimana et al., 2008; Price et al., 2009, 2010a,b; Lomma et al., 2010). The F-box domain of AnkB interacts with the host Skp1 component of the SCF1 ubiquitin ligase complex and functions as a platform for the docking of polyubiquitinated proteins to the Legionella-containing vacuolar (LCV) membrane within human cells, Acanthamoeba, and Dictyostelium discoideum (Dorer et al., 2006; Habyarimana et al., 2008; Price et al., 2009, 2010a; Al-Khodor et al., 2010a,b; Lomma et al., 2010).

In addition to hijacking the host ubiquitination machinery, prenylation of AnkB by the host cell anchors it to the membrane of the LCV and that the three host enzymes involved in prenylation are recruited to the LCV in a Dot/Icm-dependent manner, and are essential for the biological function of AnkB (Price et al., 2010b). Prenylation (farnesylation or geranylgeranylation) is a highly conserved post-translation lipid modification of eukaryotic proteins that confers hydrophobicity on the modified protein, and its targeting to membranes (Wright and Philips, 2006). Prenylation is mediated by protein geranylgeranyl transferase I (PGGT), protein farnesyl transferase (PFT), or by Rab geranylgeranyl transferase (RGGT) (Wright and Philips, 2006). Prenylated proteins 
often undergo further post-translational modifications at the ER membrane by the activity of the RCE-1 and ICMT enzymes (Wright and Philips, 2006), which cleave the terminal "-aaX” tripeptide and methylate the terminal prenylated cysteine residue, respectively. This post-translational modification plays a key role in functional activity of numerous eukaryotic proteins, including Rab proteins, Ras, G proteins, and protein kinases (Casey et al., 1989; Hancock et al., 1989; Mumby et al., 1990; Yamane et al., 1990; Wang et al., 1992). Prenylation of the AnkB effector is essential for its biological function in proliferation of L. pneumophila within the two evolutionarily distant hosts, mammalian and protozoan cells, and for intrapulmonary bacterial proliferation in the mouse model (Al-Khodor et al., 2008; Price et al., 2009).

A myriad of effectors are injected into the host cell by elaborate type III-VII translocation systems of intra-vacuolar pathogens. Although many injected bacterial effectors are anchored into the pathogen-containing vacuolar membrane or other endo-membranes, the mechanism of this membrane-anchoring is not well understood. Many intracellular bacterial pathogens capable of injecting effectors into host cells encode proteins with predicted prenylation C-terminal CaaX motif (Price et al., 2010b). Here we show that the Legionella-containing vacuole (LCV) is decorated with prenylated proteins other than AnkB. This led us to examine the genomes of L. pneumophila strains for proteins harboring the eukaryotic CaaX motif. In this study we identified 11 new C-terminal CaaX motif-containing proteins in L. pneumophila, which we designated Prenylated effectors of Legionella (PelA-K). Seven of these Pel proteins were found in L. pneumophila strain AA100/130b genome and six of these exhibited distinct cellular localization in mammalian cells that was dependent on a functional CaaX motif and the host protein prenylation machinery. Five Pel proteins were translocated into host cells by the Dot/Icm type IV translocation system of L. pneumophila and represent novel L. pneumophila effectors that contribute to evasion of lysosomal fusion by the LCV. These data show exploitation of the host posttranslational modification through prenylation by a novel set of Dot/Icm-translocated effectors of L. pneumophila that are targeted into various host membranes. It is likely that exploitation of host prenylation to anchor injected microbial effectors into various host membranes is a common theme utilized by microbes that are capable of injecting effectors into host cells.

\section{RESULTS}

IDENTIFICATION OF L. PNEUMOPHILA C-TERMINAL CAAX MOTIFCONTAINING PROTEINS

Our previous study showed that host cell prenylation of AnkB anchors it to the membrane of the LCV, and that the three host enzymes (PFTase, IcmT, and RCE-1) involved in prenylation and processing of the prenylated C-terminus are recruited to the LCV in a Dot/Icm-dependent manner and are essential for the biological function of AnkB (Price et al., 2010b). We were interested to determine if other L. pneumophila proteins were modified by the host prenylation machinery to be anchored into host membranes. To test if the LCV is decorated with other prenylated proteins apart from AnkB, we analyzed semi-purified LCVs by confocal microscopy for the presence of prenylated proteins. The U937 human macrophage cell line was infected with the WT L. pneumophila strain AA100, the ankB mutant or the ankB mutant complemented with the native $a n k B$ allele $(a n k B / c . a n k B)$ or a substitution variant allele $\left(a n k B / c \cdot a n k B{ }^{169} \mathrm{C} / \mathrm{A}\right)$ defective in prenylation. The translocation-defective isogenic $\operatorname{dot} A$ mutant was used as a control. After $2 \mathrm{~h}$ of infection, semi-purified LCVs were isolated and allowed to adhere to glass coverslips for confocal microscopy. To determine whether the farnesylated proteins were localized to the cytosolic face of the LCV membrane, which is impermeable to antibodies, the LCVs were incubated with an anti-farnesyl antibody prior to permeabilization of membranes. Following this, the LCVs were permeabilized and incubated with a monoclonal anti-Legionella antibody. Bound primary antibodies were detected using AlexaFluor conjugated secondary antibodies. The data showed that $88 \%$ of LCVs harboring the WT strain were decorated with prenylated proteins, while only $18 \%$ of LCVs harboring the type IV translocation-defective $\operatorname{dot} A$ mutant showed the presence of prenylated proteins (Figure 1). This showed that decoration of the LCV with prenylated proteins was dependent on a functional type IV secretion apparatus. Interestingly, 58\% of LCVs harboring the ankB mutant were still decorated with prenylated proteins at the LCV outer surface (Figure 1). In addition, $63 \%$ of the LCVs harboring the $a n k B$ mutant complemented with $a n k B{ }^{169} C / A$ were decorated with prenylated proteins, similar to the $a n k B$ mutant, which was significantly different for the WT strain (Student $t$-test, $p<0.01$ ) (Figure 1). The LCVs harboring the ankB mutant complemented with a WT copy of the ankB gene exhibited a frequency of decoration by prenylated proteins similar to the WT bacteria ( $85 \%$ positive) (Figure 1). Taken together, LCV membrane-anchored proteins other than AnkB at were prenylated and their presence requires a functional Dot/Icm type IV secretion. Some of the prenylated proteins that decorated the LCV were likely Dot/Icm effectors, since their presence was dependent on a function translocation system. It is also possible that some of the prenylated proteins were host proteins that were recruited by Dot/Icm effectors.

To investigate whether some of the prenylated proteins decorating the LCV were of bacterial origin we analyzed the genomes of four L. pneumophila strains (Philadelphia, Paris, Lens, and Corby) for genes encoding proteins that have a C-terminal CaaX motif similar to that found in AnkB (Price et al., 2010b). We identified a total of eleven new CaaX motif-containing proteins in strains Philadelphia, Paris, Lens, and Corby (Table 1). We designated these proteins as Prenylated effectors of Legionella (PelA-K). Of these 11, 5 [PelA, B, E (LegG1), H, and I (PepO)] are shared between all the sequenced strains. PelD is found in Lens, Paris, and Corby, while PelF is found in Philadelphia, Paris, and Corby. PelC (LegC1) is found only in Philadelphia, while PelJ and $\mathrm{K}$ are only found in Lens. Interestingly, PelB in strain Lens has a mutation that resulted in substitution of the crucial cysteine residue in the CaaX motif to alanine. In addition, PelD in strains Lens and Corby has a mutation resulting in substitution of the crucial cysteine residue of the CaaX motif to a tyrosine.

\section{DISTINCT LOCALIZATION OF ECTOPICALLY EXPRESSED PEL PROTEINS IN MAMMALIAN CELLS}

By using PCR we identified that L. pneumophilaAA100 also harbored PelA, B, D, E, F, H, and I., the Pel proteins that are shared amongst the sequenced strains (Table 1). Our previous work showed that 


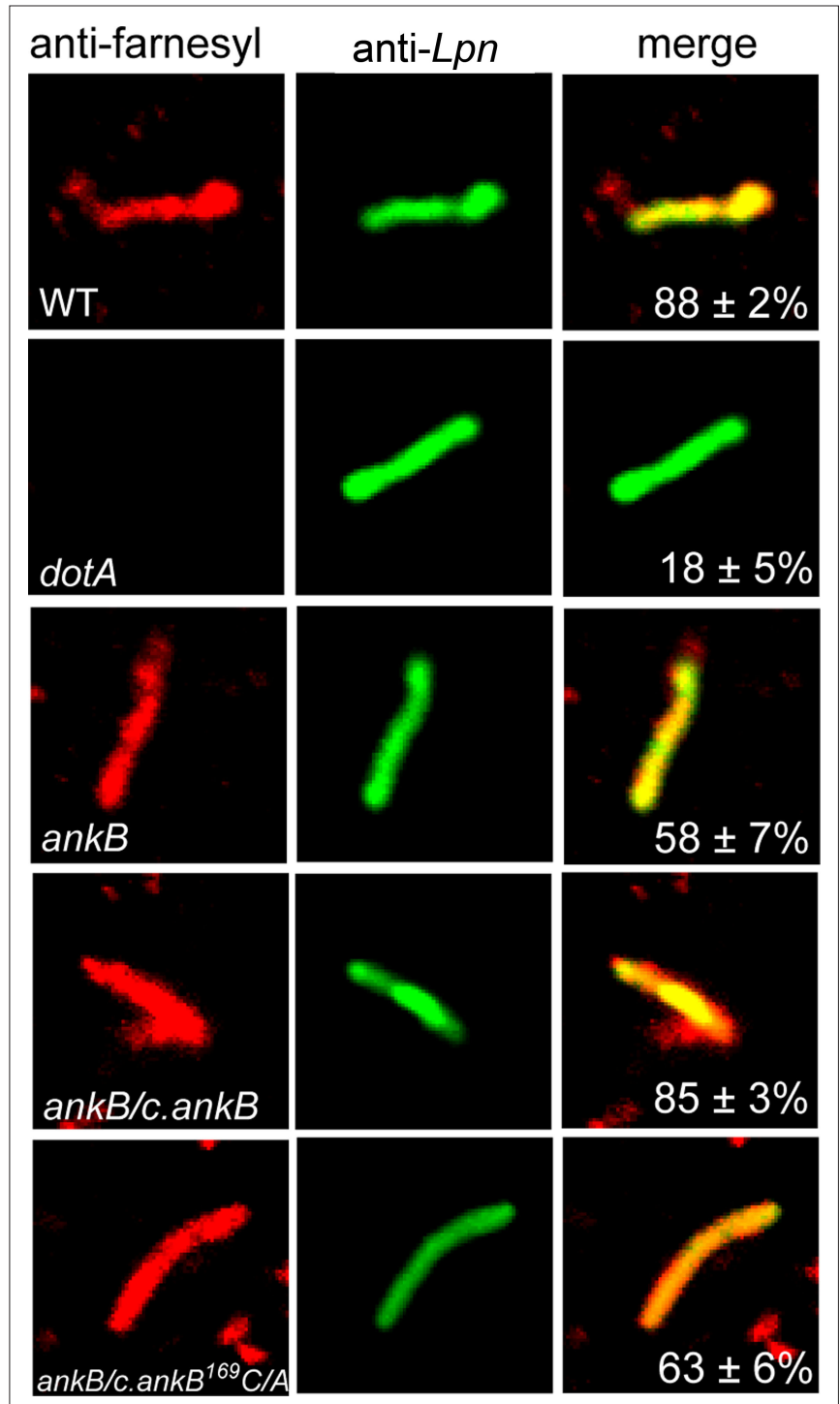

FIGURE 1 |The LCV membrane is decorated with prenylated proteins in a Dot/Icm-dependent manner. Infected $U 937$ cells were lysed after $2 \mathrm{~h}$ infection by the WT, dotA, ankB, ankB/C.ankB or ankB/C.ankB ${ }^{169} \mathrm{C} / \mathrm{A}$ bacteria. The LCVs were isolated and were probed with anti-farnesyl antiserum prior to permeabilization (red) and examine by confocal microscopy to determine whether the prenylated proteins were localized to the cytosolic side of the LCV membrane. After permeabilization, the LCVs were probed with mouse anti-L. pneumophila (Lpn) monoclonal antibodies (green) to visualize the bacteria within the LCV. Quantitation is shown in the merged panels, where the numbers represents percentage plus standard deviation of LCVs that bound anti-farnesyl antiserum prior to permeabilization. Analyses were based on examination of $100 \mathrm{LCV}$ s from triplicate samples. All experiments were performed in triplicate and representative examples are shown. All the results in this figure are representative of three independent experiments.

ectopically expressed 3X-FLAG AnkB in HEK293 cells exhibits a striking localization at the cell periphery (Price et al., 2009) and this localization is dependent on prenylation of the C-terminal CaaX motif of AnkB (Price et al., 2010b). To determine if the Pel proteins also exhibit distinct membrane localization in mammalian cells we generated $3 \mathrm{X}$-Flag fusions to the $\mathrm{N}$-terminus of these proteins, and transfected HEK293 cells. Following 24 h of transfection, HEK293 cells were fixed and permeabilized and incubated with anti-Flag antibody and examined by confocal microscopy (Figure 2A). The 3X-Flag AnkB control exhibited distinct localization to the cell plasma membrane as expected (Figure 2A). Interestingly all the Pel proteins were not diffusely distributed throughout the cytosol but showed distinct localization in the cell (Figure 2A). 3X-Flag PelA concentrated around the periphery of enlarged vesicles and as distinct punctate spots within the cell (Figure 2A). 3X-Flag PelB localized as intense punctate spots in HEK293 cells, indicating vesicular localization (Figure 2A). 3X-Flag PelD, E and F localized similarly in HEK293 cells, as punctate spots and weakly around the cell periphery (Figure 2A). 3X-Flag PelH localized strongly around the cell periphery and as punctate spots in the cell, reminiscent of that observed for 3X-Flag AnkB (Figure 2A). This shows that ectopically expressed Pel proteins are targeted to distinct locations within mammalian cells. We could not detect ectopic expression of 3X-Flag PelI (PepO), which may be due to potentially detrimental effects of over-expressing a metalloprotease in a cell.

\section{SPECIFIC LOCALIZATION OF THE PELS IN MAMMALIAN CELLS THROUGH HOST-MEDIATED PRENYLATION OF THE C-TERMINAL CAAX MOTIF}

We utilized two independent and complementary approaches to determine whether the distinct localization of the Pel proteins in mammalian cells was due to host-mediated prenylation, resulting in association of these proteins with cellular membranes. Our first approach was to inhibit the host prenylation machinery to examine whether that would alter localization of the Pel proteins in the host cell. To address this, HEK293 cells were pre-treated with the prenylation inhibitor FTI-277 (Lerner et al., 1995) and then transfected with the 3X-FLAG Pel constructs, and analyzed by confocal microscopy (Figure 2B). As expected, the 3X-Flag AnkB control was no longer localized to the plasma membrane in cells treated with FTI-277 and was found throughout the cytosol (Figures 2A,B). Interestingly, all the Pel proteins exhibited a shift in cellular localization following chemical inhibition of PFTase (Figure 2B). The 3X-Flag PelA in prenylation-inhibited cells was no longer found around enlarged vacuoles but became more evenly distributed throughout the cytosol (Figures 2A,B). The distribution of 3X-Flag $\mathrm{PelB}$, which is found as distinct punctate spots in untreated cells, completely shifted to a more homogenous cytosolic distribution in prenylation-inhibited cells (Figures 2A,B). The 3X-Flag PelD, E and $\mathrm{F}$ which were localized, in untreated cells, to small punctate spots throughout the cytoplasm and on the plasma membrane exhibited an even distribution in the cytosol in prenylation-inhibited cells (Figures 2A,B). Localization of 3X-Flag PelH, which exhibits similar plasma membrane localization to 3X-Flag AnkB, was completely altered to a cytoplasmic distribution in prenylation-inhibited cells (Figures 2A,B). These data show that the specific localization of the Pel proteins when expressed in HEK293 cells is dependent on the activity of the host prenylation machinery.

Our second approach to determine whether localization of the Pel proteins to distinct cellular locations was directly due to prenylation modification was a genetic approach from the microbe side. The conserved cysteine residue in the C-terminal CaaX motif of each Pel protein was substituted by alanine. We have previously shown that mutation of the conserved cysteine residue of the 
CaaX motif of AnkB abolish prenylation and anchoring of AnkB to the host plasma membrane (Price et al., 2010b). Examination by confocal microscopy showed that ectopically expressed 3X-FLAG
AnkB ${ }^{169} \mathrm{C} / \mathrm{A}$ control was distributed evenly throughout the cytosol in HEK293 cells, as expected (Figures 3A,B). Interestingly, mutation of the conserved cysteine residue in the CaaX motif of all the Pel

Table 1 | Genes in the four sequenced L. pneumophila genomes that harbor a C-terminal CaaX motif. Genes were identified using the Legiolist website (http://genolist.pasteur.fr/LegioList/) and the "search pattern" tool.

\begin{tabular}{|c|c|c|c|c|c|c|c|}
\hline Gene name & Philadelphia & Lens & Paris & Corby & CaaX motif & Gene designation & Domains \\
\hline PelB & Ipg0770 & $|\mathrm{p}| 0811^{*}$ & Ipp0835 & Ipc2522 & CLIK & Hypothetical & - \\
\hline PelD & - & $|p| 1858 * *$ & Ipp1863 & Ipc1344** & CSLL & Hypothetical & - \\
\hline PelE & lpg1976 & |p|1953 & Ipp1959 & Ipc1462 & CNLL & LegG1 & ATS1 \\
\hline PelG & lpg2525 & - & - & - & CSIL & Hypothetical & - \\
\hline PelH & lpg2541 & $|p| 2462$ & Ipp2607 & Ipc1927 & CTIM & Hypothetical & - \\
\hline Pell & lpg2607 & |p|2530 & Ipp2660 & Ipc0534 & CIIW & PepO & Peptidase M13 \\
\hline PelJ & - & |p|2477 & - & - & CTIM & Hypothetical & HAD-SF-IIIC \\
\hline PelK & - & |p|2806 & - & - & CVIS & Hypothetical & - \\
\hline
\end{tabular}

*Lens CaaX motif is mutated to ALAK, **Lens and Corby CaaX motif is missing the conserved cysteine residue (YSLL), ***Paris 2082 has a frame shift mutation resulting in an 18 amino acid truncation at the C-terminus, abolishing the CaaX motif. AnkB is only known translocated CaaX motif-containing protein.
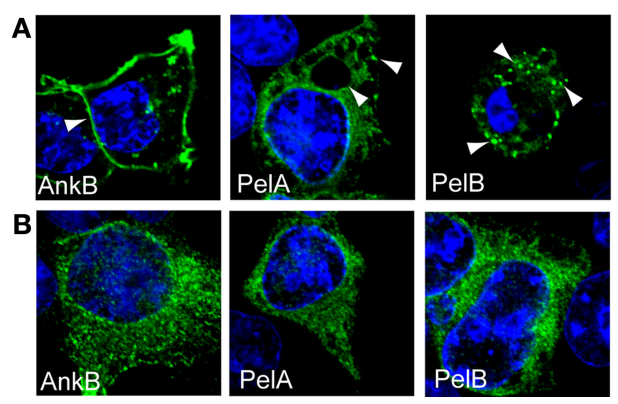

FIGURE 2 | Cellular distribution of ectopically expressed 3X-FLAG Pel proteins is dependent on host prenylation machinery. Representative confocal microscopy images of untreated (A) or FTI-277-treated (B) HEK293 cells ectopically expressing 3X-FLAG tagged AnkB, PelA, PelB, PelD, PelE, PelF or PelH
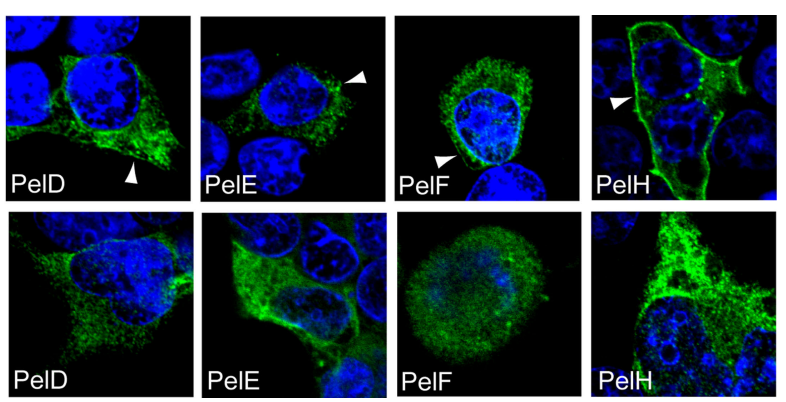

fusion proteins. Green indicates labeling with anti-FLAG M2 antibody and the nucleus is stained blue (DAPI). The arrowheads indicate strong localization of the various $3 \mathrm{X}$-Flag fusions at distinct cellular locations. All experiments were performed three times and representative examples are shown.
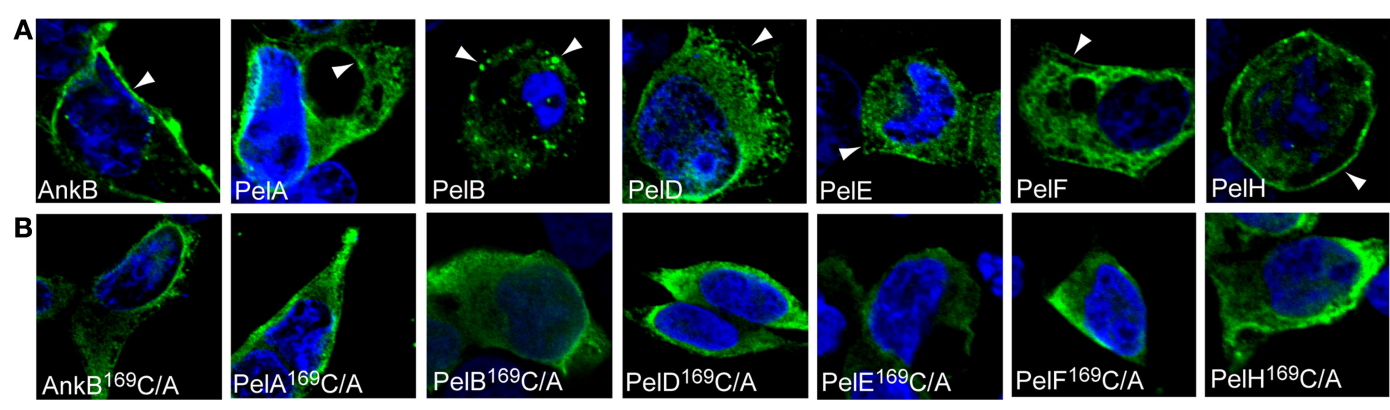

FIGURE 3 |The CaaX motifs of the Pels are indispensable for their distinct cellular distribution. (A) Representative confocal microscopy images of HEK293 cells ectopically expressing 3X-FLAG tagged AnkB, PelA, PelB, PelD, PelE, PelF or PelH fusion proteins. Green indicates labeling with anti-FLAG M2 antibody and the nucleus is stained blue (DAPI). The arrowheads indicate strong

localization of the various 3X-Flag fusions at distinct cellular locations. (B) Representative confocal microscopy images of HEK293 cells ectopically expressing 3X-FLAG tagged AnkB ${ }^{169} \mathrm{C} / \mathrm{A}$, PelA ${ }^{169} \mathrm{C} / \mathrm{A}$, PelB ${ }^{169} \mathrm{C} / \mathrm{A}$, PelD ${ }^{169} \mathrm{C} / \mathrm{A}$, PelE ${ }^{169} \mathrm{C} / \mathrm{A}$, PelF ${ }^{169} \mathrm{C} / \mathrm{A}$ or $\mathrm{PelH}{ }^{169} \mathrm{C} / \mathrm{A}$ fusion proteins. All experiments were performed three times and representative examples are shown. 
proteins resulted in a re-distribution of these proteins throughout the cytosol, similar to the results obtained upon chemical inhibition of prenylation (Figures 3A,B). This clearly shows that the cysteine residue is crucial in the localization of the Pel proteins to distinct cellular locations, and these are likely to be various host membranes. Taken together, these data indicate that the Pel proteins are modified by the host prenylation machinery that enables their targeting to specific cellular location, which is most likely various host membranes.

\section{TRANSLOCATION OF PEL PROTEINS INTO MACROPHAGES}

To be prenylated by the host machinery, the CaaX motif-containing Pel proteins must be translocated out of the LCV. To examine whether the Pel proteins were translocated into host cells by the Dot/Icm type IV translocation system of L. pneumophila, we utilized the calmodulin-dependent adenylate cyclase reporter fusion assay using ELISA (Sory and Cornelis, 1994; Al-Khodor et al., 2008). The U937 cells were infected for $1 \mathrm{~h}$ with the WT strain harboring the vector alone, ralF-CyaA and ankB-CyaA as positive controls, or Pel-CyaA fusions. The translocation-defective dotA mutant harboring the various CyaA fusions was used to determine whether translocation was Dot/Icm-dependent. The data showed that PelA, B, D, E, F, and I were translocated into the host cell cytosol similar to AnkB and the RalF effector controls (Figure 4). Translocation of PelA and PelB was reproducibly lower compared to the controls and other Pel proteins. However, translocation of both PelA and PelB was higher compared to the empty vector negative control, was Dot/ Icm-dependent and this was statistically significant (Student $t$-test, $p<0.01)$. Furthermore, translocation of all the Pel proteins was not observed in the translocation-defective $\operatorname{dot} A$ mutant, indicating

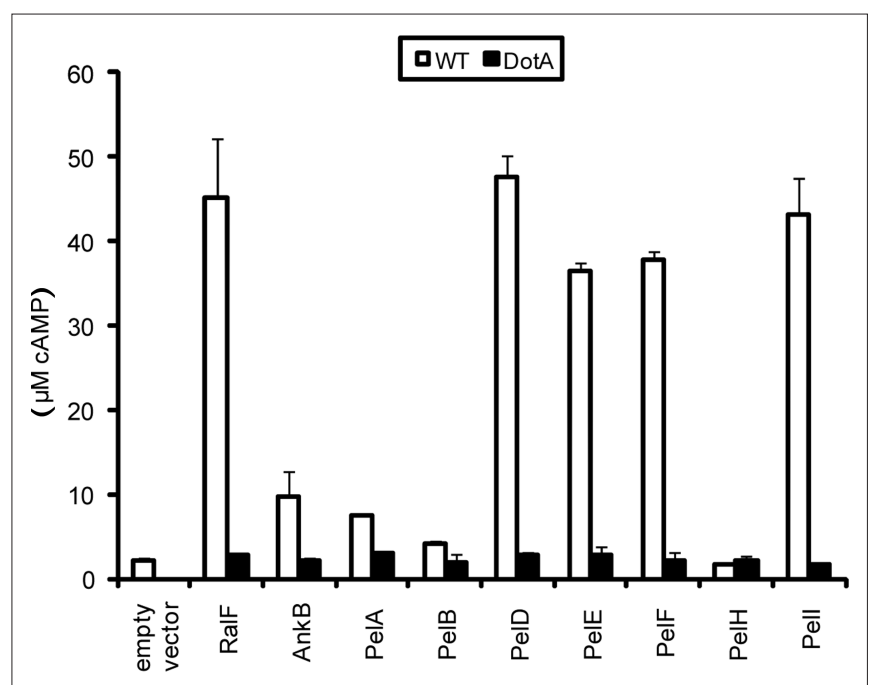

FIGURE 4 | Dot/lcm-dependent injection of the Pel proteins of L. pneumophila into human macrophages. Translocation of the Pels into U937 cells was determined at $1 \mathrm{~h}$ post-infection. WT or dotA mutant bacteria harbored either empty vector (pCya) or Cya hybrids of RalF, AnkB or PelA, PelB, PelD, PelE, PelF, PelH or Pell. All experiments were performed three times and representative examples are shown. The data are the mean of triplicate samples and the error bars are the standard deviations. Translocation of all Pel proteins was statistically significant compared to bacteria harboring empty vector (Students $t$-test, $p$-value $<0.01$ ). that translocation of the Pel proteins was Dot/Icm-dependent. PelH was not translocated using this assay, which may be due to interference by the Cya fusion. Interestingly, when ectopically expressed in cells, this protein exhibited similar localization to AnkB. Further studies are required to rule out the possibility PelH is not translocated. These data show that L. pneumophila strain AA100/130b harbors a group of novel translocated effectors, in addition to AnkB, that are modified by the host prenylation machinery that anchors them into host membranes.

\section{EFFECT OF HOST PRENYLATION OF TRAFFICKING OF THE LCV}

Upon phagocytosis L. pneumophila evades endocytic fusion and intercepts ER-to-Golgi vesicle traffic to remodel its phagosome into an ER-derived vacuole, which is essential for intra-vacuolar proliferation (Kagan and Roy, 2002; Molmeret et al., 2005; Shin and Roy, 2008; Isberg et al., 2009). Since inhibition of the host prenylation machinery blocks intracellular proliferation of $L$. pneumophila, we determined whether that was due to alteration in trafficking of the LCV by examination of colocalization of the LCV with the late endosomal/lysosomal marker Lamp2 and the lysosomal enzyme Cathepsin D. The U937 cells were pre-treated with prenylation inhibitor and then infected with WT or formalin-killed WT bacteria. Following $2 \mathrm{~h}$ of infection, fixed cells were incubated with anti-Legionella and anti-Lamp2 or anti-cathepsin $\mathrm{D}$ antibody and analyzed by confocal microscopy. The data showed that LCVs harboring the WT bacteria did not localize with both Lamp2 and Cathepsin D staining in untreated U937 cells (28 and $26 \%$ localization respectively), indicating WT bacteria evaded endocytic fusion (Figures 5A,B). In prenylation-inhibited cells, there was a moderate increase in WT bacteria co-localizing with both Lamp2 and Cathepsin D (35\% and 37\% positive, respectively (Figures 5A,B) and this was statistically significant (Student $t$-test, $p<0.05)$. Over $83 \%$ of formalin-killed WT bacteria localized with Lamp2 or cathepsin D in untreated or prenylation-inhibited cells (Figures 5A,B) showing that inhibition of the prenylation machinery did not affect the activity of endosomal-lysosomal pathway in the cell. Taken together, these data show that host prenylation machinery contributes to the ability of living L. pneumophila to evade endocytic fusion.

\section{DISCUSSION}

Although numerous bacterial effectors injected by various intravacuolar pathogens are localized to the pathogen-containing vacuolar membrane and other host membranes, the mechanisms of this localization are not well known. We have recently shown that host-mediated prenylation of the AnkB effector of L. pneumophila represents a new paradigm for anchoring microbial effectors to the pathogen-containing vacuolar membrane (Price et al., 2010b). The presence of putative $\mathrm{CaaX}$ motif proteins in $>20$ species of extracellular and intracellular bacterial pathogens of mammals and plants and endosymbionts that are capable of injecting effectors into host cell suggests that hijacking the host prenylation pathway may be a common theme utilized by microbial pathogens that inject effectors into host cells (Price et al., 2010b). Prior to this study, the AnkB F-box effector was the only microbial translocated effector known to be modified by the host prenylation machinery where this host-mediated post-translational modification was essential 

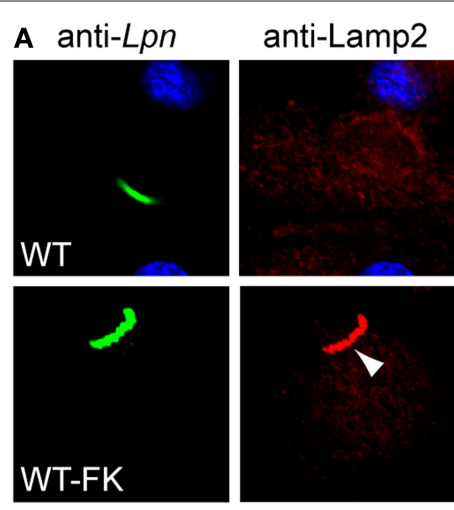

FTI-277
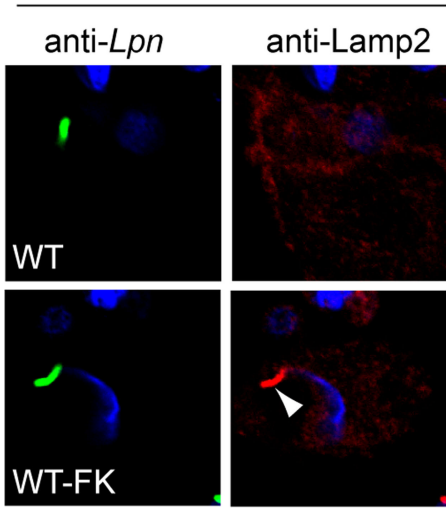

merge
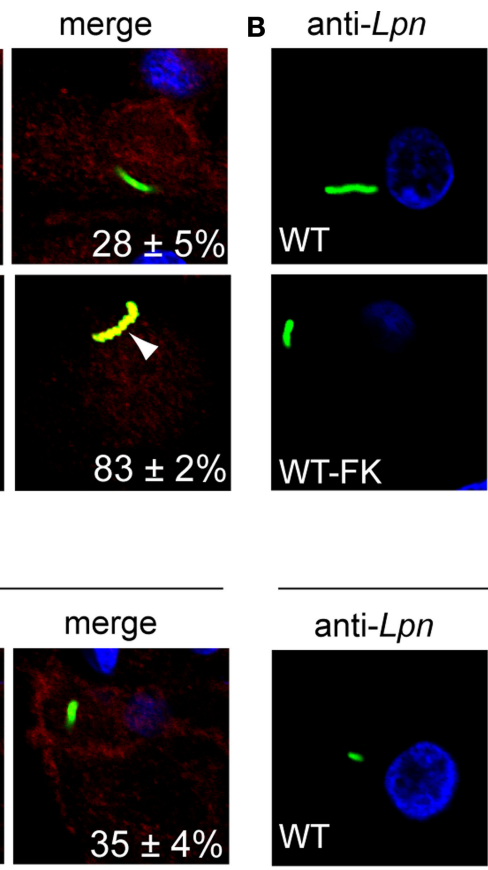

$83 \pm 2 \%$

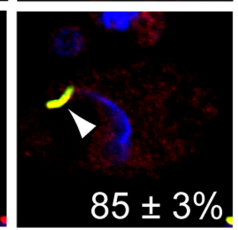

FIGURE 5 | Trafficking of the LCV in prenylation-inhibited U937 cells. (A,B) Untreated or FTI-277-treated U937 cells infected for $1 \mathrm{~h}$ with WT or WT formalin-killed bacteria (WT-FK). After $2 \mathrm{~h}$ of infection, the cells were labeled with anti-Lamp2 (red) or anti-cathepsin D (CathD; red) antibodies and analyzed by confocal microscopy. Arrowheads indicate colocalization of the LCVs with LAMP-2 or cathepsin D. The formalin-killedWT were used as a positive control for Lamp2 and cathepsin D colocalization. Quantitation is shown in the merged

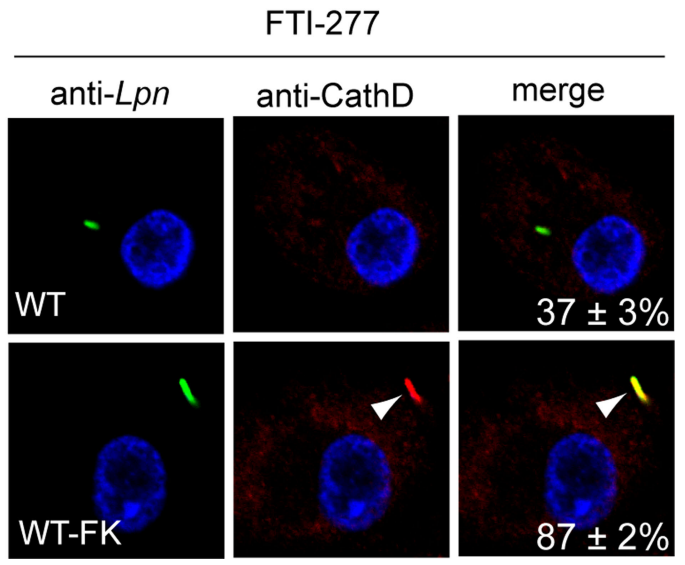

for intra-vacuolar proliferation of the pathogen (Al-Khodor et al., 2008, 2010a,b; Habyarimana et al., 2008; Price et al., 2009, 2010a,b). This study has significantly expanded the list of known translocated effectors modified by the host prenylation machinery. The only other confirmed bacterial effector previously shown to be prenylated is SifA of S. typhimurium (Boucrot et al., 2003; Reinicke et al., 2005). SifA is translocated by the type III secretion system and is localized to the Salmonella-induced filaments that connect Salmonella-containing vacuoles, but its biological function is not known (Boucrot et al., 2003; Reinicke et al., 2005). Prenylation of SifA by PGGT is dispensable for its biological function, since a prenylation-defective sifA substitution mutant is fully competent in intracellular proliferation and virulence in the mouse model (Reinicke et al., 2005). In contrast, prenylation of AnkB is essential for its biological function in vitro and for intrapulmonary proliferation in the mice model of Legionnaires' disease (Price et al., 2010b).

Our data show that L. pneumophila translocates several novel effectors that are modified by the host prenylation machinery, similar to AnkB (Price et al., 2010b). Our data have clearly shown panels, where the numbers represent the percentage of LCVs that colocalized with the respective marker. Analyses were based on the examination of 100 infected cells from multiple coverslips. Data represent means \pm standard deviation. Results are representative of three independent experiments performed in triplicate. Lpn, L. pneumophila. Increased localization of WT LCVs to Lamp2 and cathepsin D in FTi-277 treated cells was statistically significant (Students t-test, $p$-value $<0.05$ ). that the Pel effectors exhibit distinct localization in the cells and the host-mediated prenylation of the CaaX motif was essential for this localization, which is most likely various host membranes. Currently the cellular localization and function of the Pel proteins during infection of macrophages is unknown, but studies are underway to investigate these questions. It is possible that the Pel proteins target to the LCV membrane similar to AnkB (Price et al., 2010b). When ectopically expressed in mammalian cells, 3X-Flag AnkB localizes to the inner leaflet of the plasma membrane, but native AnkB translocated by bacteria during infection is found solely on the cytosolic face of the LCV membrane (Price et al., 2010b). We have shown that PFTase, IcmT, and RCE-1 are all recruited to the LCV in a Dot/Icm-dependent manner (Segal et al., 1998; Vogel et al., 1998; Price et al., 2010a). Prenylated proteins often undergo further post-translational modifications at the ER membrane by the activity of the RCE- 1 and ICMT enzymes (Wright and Philips, 2006), which cleave the terminal "-aaX" tri-peptide and methylate the terminal prenylated cysteine residue, respectively. RCE-1 and ICMT are localized to the ER-derived LCV membrane in a Dot/Icm-dependent manner (Price et al., 2010b). The presence of 
PFTase, RCE-1 and IcmT at the LCV membrane may enable local prenylation of Pel proteins immediately following translocation. It is likely that some of the Pels integrate into the outer leaflet of the LCV membrane, similar to what we have observed with AnkB (Price et al., 2010b) and may account for the presence of prenylated proteins other than AnkB at the LCV surface. It is also possible that some of the prenylated proteins decorating the LCV are host proteins that are recruited to the LCV by Dot/Icm-translocated effectors, and prenylation of these host proteins may occur locally at the LCV membrane.

It is important to note that the exact nature of the prenylation modification (farnesylation or geranylgeranylation) of the Pel proteins is currently unknown. The inhibitor used in this study, FTI-277 is a peptidomimetic inhibitor of PFTase but also exhibits cross-inhibition of PGGT (Lerner et al., 1995). Therefore, we cannot determine whether the Pels are geranylgeranylated or farnesylated. Interestingly, mammalian K-Ras and N-Ras are preferentially farnesylated, however when PFTase is inhibited, these two proteins can still be geranylgeranylated by PGGT and be functional (James et al., 1995; Whyte et al., 1997; Geryk-Hall et al., 2010). It will be interesting to determine if this phenomenon also occurs in Pel prenylation. Since inhibition of prenylation blocks intra-vacuolar replication of L. pneumophila and related Legionella species (Price et al., 2010b), understanding the exact lipid modifications of prenylated effectors will be critical in understanding how host-mediated prenylation of bacterial effectors contributes to bacterial replication, which may facilitate the development of novel anti-Legionella therapeutics.

Avoidance of the endocytic pathway is crucial for the success of L. pneumophila to replicate in macrophages and in ameba (Kagan and Roy, 2002; Molmeret et al., 2005; Shin and Roy, 2008; Isberg et al., 2009). We have shown that blocking host prenylation activity results in reduced ability of L. pneumophila to avoid trafficking to the lysosome. Membrane bound prenylated AnkB is unlikely to play a role in intracellular trafficking of the $\mathrm{LCV}$, since the ankB null bacteria avoid trafficking to the lysosome similar to WT bacteria (Al-Khodor et al., 2008). However the roles of the Pel proteins are unknown. Perhaps when integrated into membranes, these prenylated proteins may directly assist in evading the endocytic pathway, thus contribute to the ability of L. pneumophila to establish a safe replicative niche. It is important to note that contribution of the Pels to evasion of the lysosomal pathway by the LCV is very modest. Therefore, other more important non-prenylated effectors play the major role in evasion of the endosomal-lysosomal pathway by the LCV. We also cannot rule out the possibility that prenylated host proteins, hijacked by L. pneumophila, also contribute to evasion of the endocytic pathway by the LCV. The function of the Pels and potential prenylated host proteins in remodeling the LCV into a proliferative niche represents exciting new avenues of research.

Manipulation of host activities is crucial for the success of intracellular pathogens. This is often achieved by translocation of effectors that harbor eukaryotic protein domains that mimic the function of host proteins (Franco et al., 2009; Galan, 2009; Hauser, 2009; Ibarra and Steele-Mortimer, 2009; Isberg et al., 2009). We show that this intimate host/pathogen relationship goes even further with the ability of microbes to translocate effectors that take advantage of host post-translational modification such as prenylation, to enable the correct cellular localization of effectors. Given the abundance of CaaX motif-containing proteins in L. pneumophila and putative CaaX-containing proteins in other extracellular and intracellular pathogens of mammals and plants such as Mycobacterium, Salmonella, Anaplasma, Bartonella, Brucella, Agrobacterium, and Toxoplasma (Price et al., 2010b), it is likely that the ability to exploit the host prenylation machinery may be a common theme utilized by microbial pathogens and endosymbionts capable of injecting effectors in host cells to anchor the injected effectors into specific host membranes.

\section{MATERIALS AND METHODS BACTERIAL STRAINS, CELL CULTURES, AND INFECTIONS}

Legionella pneumophila strain AA100/130b (ATCC BAA-74) and the isogenic mutants $\operatorname{dot} A, a n k B$, and complemented ankB mutants were grown as described previously (Al-Khodor et al., 2008). Maintenance of U937 and HEK293 cells were performed as previously described (Price et al., 2009). Infection studies for purification of the LCVs and effector translocation were performed as we described previously (Al-Khodor et al., 2008; Price et al., 2009). Briefly, macrophages were infected at a multiplicity of infection (MOI) of 10 (LCVs) or 50 (Cya assay). Measurement of cAMP in cell lysates for adenylate cyclase fusion assays was performed using the Direct Cyclic AMP Enzyme Immunoassay kit (Assay Designs), as we described previously (Al-Khodor et al., 2008). The Student $t$-test was used to determine if differences in cAMP levels were significant in strains harboring pCYA-Pel fusions compared to the strain harboring the empty vector and whether translocation was Dot/Icm-dependent. Purification of post-nuclear supernatant containing LCVs was performed as previously described (Price et al., 2010b). For analysis of LCV trafficking, U937 cells on glass coverslips were infected with WT or formalin-killed bacteria for $1 \mathrm{~h}$ followed by treatment with $50 \mu \mathrm{g} / \mathrm{ml}$ gentamicin for $1 \mathrm{~h}$ to kill extracellular bacteria. Infected cells were then fixed and permeabilized with ice cold methanol for $5 \mathrm{~min}$.

\section{TRANSFECTIONS AND INHIBITORS}

Cloning of the 3X-FLAG tagged Pel alleles was performed as described previously (Price et al., 2009), using specific primers (Table A1 in Appendix). Mutations in the CaaX motif were performed as described previously (Price et al., 2010b), using specific primers (Table A1 in Appendix). HEK293 cells were transfected using Fugene HD reagent (Roche, Mannheim), as we described previously (Price et al., 2009). The inhibitor FTI-277 (Calbiochem, Gibbstown, NJ) was re-suspended in DMSO +0.4 mM DTT and used immediately, and maintained in the growth media throughout the experiment.

\section{CONFOCAL LASER SCANNING MICROSCOPY}

Processing of infected cells for confocal microscopy was performed as we described previously (Price et al., 2009). Polyclonal rabbit anti-L. pneumophila antiserum and monoclonal mouse anti- $L$. pneumophila antibodies were detected by Alexa-Fluor 488-conjugated donkey anti-rabbit or -mouse IgG (Invitrogen, Carlsbad, CA, USA). Prenylation was detected with a rabbit anti-farnesyl antibody (1/50 dilution) (Abcam, Cambridge, MA, USA) followed by Alexa-Fluor 555-conjugated donkey anti-rabbit IgG (Invitrogen, Carlsbad, CA, USA) (Price et al., 2010b). Mouse anti-Lamp2 
and mouse anti-cathepsin $\mathrm{D}$ antiserum was used as previously described (Al-Khodor et al., 2008). Alexa-fluor tagged antibodies against mouse IgG were used as secondary antibodies (Invitrogen, Carlsbad, CA, USA). 3X-flag fusion proteins were detected using anti-Flag M2 antibody (Sigma, St Louis, MO, USA) and Alexa-fluor conjugated antibodies. The cells were examined with an Olympus FV1000 laser scanning confocal microscope as we described previously (Price et al., 2009). On average, 8-15 $0.2 \mu \mathrm{m}$ serial $Z$ sections of each image were captured and stored for further analyses, using

\section{REFERENCES}

Al-Khodor, S., Al-Quadan, T., and Abu Kwaik, Y. (2010a). Temporal and differential regulation of expression of the eukaryotic-like ankyrin effectors of L. pneumophila. Environ. Microbiol. Rep. 2, 677-684.

Al-Khodor, S., Price, C. T., Kalia, A., and Abu Kwaik, Y. (2010b). Functional diversity of ankyrin repeats in microbial proteins. Trends Microbiol. 18, 132-139.

Al-Khodor, S., Price, C. T., Habyarimana, F., Kalia, A., and Abu Kwaik, Y. (2008). A Dot/Icm-translocated ankyrin protein of Legionella pneumophila is required for intracellular proliferation within human macrophages and protozoa. Mol. Microbiol. 70, 908-923.

Boucrot, E., Beuzon, C. R., Holden, D. W., Gorvel, J. P., and Meresse, S. (2003). Salmonella typhimurium SifA effector protein requires its membraneanchoring C-terminal hexapeptide for its biological function. J. Biol. Chem. 278, 14196-14202.

Casey, P. J., Solski, P.A., Der, C. J., and Buss, J. E. (1989). p21ras is modified by a farnesyl isoprenoid. Proc. Natl. Acad. Sci. U.S.A. 86, 8323-8327.

de Felipe, K. S., Glover, R. T., Charpentier, X., Anderson, O. R., Reyes, M., Pericone, C. D., and Shuman, H. A. (2008).Legionella eukaryotic-like type IV substrates interfere with organelle trafficking. PLoS Pathog. 4, e1000117. doi: 10.1371/journal.ppat.1000117.

Dorer, M. S., Kirton, D., Bader, J. S., and Isberg, R. R. (2006). RNA interference analysis of Legionella in Drosophila cells: exploitation of early secretory apparatus dynamics. PLoS Pathog. 2, e34. doi: 10.1371/journal.ppat.0020034.

Franco, I. S., Shuman, H. A., and Charpentier, X. (2009). The perplexing functions and surprising origins of Legionella pneumophila type IV secretion effectors. Cell. Microbiol. $11,1435-1443$.

Galan,J.E. (2009). Common themes in the design and function of bacterial effectors. Cell Host Microbe 5, 571-579.

Geryk-Hall, M., Yang, Y., and Hughes, D. P. (2010). Driven to death: inhibition of farnesylation increases ras activity in osteosarcoma and promotes growth arrest and cell death. Mol. Cancer Ther. 9, 1111-1119.

Habyarimana, F., Al-Khodor, S., Kalia, A., Graham, J. E., Price, C. T., Garcia, M. T., and Kwaik, Y. A. (2008). Role for the Ankyrin eukaryotic-like genes of Legionella pneumophila in parasitism of protozoan hosts and human macrophages. Environ. Microbiol. 10, 1460-1474.

Hancock, J. F., Magee, A. I., Childs, J. E., and Marshall, C. J. (1989). All ras proteins are polyisoprenylated but only some are palmitoylated. Cell 57, 1167-1177.

Hauser, A. R. (2009). The type III secretion system of Pseudomonas aeruginosa: infection by injection. Nat. Rev. Microbiol. 7, 654-665.

Ibarra, J. A., and Steele-Mortimer, O. (2009). Salmonella-the ultimate insider. Salmonella virulence factors that modulate intracellular survival. Cell Microbiol. 11, 1579-1586.

Isberg, R. R., O'Connor, T. J., and Heidtman, M. (2009). The Legionella pneumophila replication vacuole: making a cosy niche inside host cells. Nat. Rev. Microbiol. 7, 13-24.

James, G. L., Goldstein, J. L., and Brown, M. S. (1995). Polylysine and CVIM sequences of K-RasB dictate specificity of prenylation and confer resistance to benzodiazepine peptidomimetic in vitro. J. Biol. Chem. 270, 6221-6226.

Kagan,J.C., and Roy, C.R. (2002). Legionella phagosomes intercept vesicular traffic from endoplasmic reticulum exit sites. Nat. Cell Biol. 4, 945-954.

Lerner, E. C., Qian, Y., Blaskovich, M. A., Fossum, R. D., Vogt, A., Sun, J., Cox, A. D., Der, C. J., Hamilton, A. D., and Sebti, S. M. (1995). Ras CAAX peptidomimetic FTI-277 selectively blocks oncogenic Ras signaling by inducing cytoplasmic accumulation of inactive Ras-Raf complexes. J. Biol. Chem. 270, 26802-26806.

Lomma, M., Dervins-Ravault, D., Rolando, M., Nora, T., Newton, H. J., Sansom, F. M., Sahr, T., Gomez-Valero, L., Jules, M., Hartland, E. L., and Buchrieser, C. (2010). The Legionella pneumophila F-box protein Lpp2082 (AnkB) modulates ubiquitination of the host protein parvin B and pro-

Adobe Photoshop CS3. The Student $t$-test was used to determine if differences in localization of LCV with Lamp2 and CathD were statistically significant.

\section{ACKNOWLEDGMENTS}

Yousef Abu Kwaik is supported by Public Health Service Awards R01AI43965 and R01AI069321 from NIAID and by the commonwealth of Kentucky Research Challenge Trust Fund. The authors declare no competing financial interests.

motes intracellular replication. Cell. Microbiol. 12, 1272-1291.

Molmeret, M., Horn, M., Wagner, M., Santic, M., and Abu Kwaik, Y. (2005). Amoebae as training grounds for intracellular bacterial pathogens. Appl. Environ. Microbiol. 71, 20-28.

Mumby, S. M., Casey, P. J., Gilman, A. G., Gutowski, S., and Sternweis, P. C. (1990). G protein gamma subunits contain a 20-carbon isoprenoid. Proc. Natl. Acad. Sci. U.S.A. 87, 5873-5877.

Price, C. T., Al-Khodor, S., Al-Quadan, T., and Abu Kwaik, Y. (2010a). Indispensable role for the eukaryoticlike ankyrin domains of the Ankyrin B effector of Legionella pneumophila within macrophages and amoebae. Infect. Immun. 78, 2079-2088.

Price, C. T., Al-Quadan, T., Santic, M., Jones, S. C., and Abu Kwaik, Y. (2010b). Exploitation of conserved eukaryotic host cell farnesylation machinery by an F-box effector of Legionella pneumophila. J. Exp. Med. 207, 1713-1726.

Price, C. T., Al-Khodor, S., Al-Quadan, T., Santic, M., Habyarimana, F., Kalia, A., and Kwaik, Y. A. (2009). Molecular mimicry by an F-box effector of Legionella pneumophila hijacks a conserved polyubiquitination machinery within macrophages and protozoa. PLoS Pathog. 5, e1000704. doi: 10.1371/journal.ppat.1000704.

Reinicke, A. T., Hutchinson, J. L., Magee, A. I., Mastroeni, P., Trowsdale, J., and Kelly, A. P. (2005). A Salmonella typhimurium effector protein SifA is modified by host cell prenylation and S-acylation machinery. J. Biol. Chem. 280, 14620-14627.

Segal, G., Purcell, M., and Shuman, H. A. (1998). Host cell killing and bacterial conjugation require overlapping sets of genes within a $22-\mathrm{kb}$ region of the Legionella pneumophila genome. Proc. Natl. Acad. Sci. U.S.A. 95, 1669-1674.

Shin, S., and Roy, C. R. (2008). Host cell processes that influence the intracellular survival of Legionella pneumophila. Cell. Microbiol. 10, 1209-1220.

Sory, M. P., and Cornelis, G. R. (1994). Translocation of a hybrid YopEadenylate cyclase from Yersinia enterocolitica into HeLa cells. Mol. Microbiol. 14, 583-594.
Vogel, J.P., Andrews, H. L., Wong, S. K., and Isberg, R. R. (1998). Conjugative transfer by the virulence system of Legionella pneumophila. Science 279, 873-876.

Wang, P. C., Vancura, A., Mitcheson, T. G., and Kuret, J. (1992). Two genes in Saccharomyces cerevisiae encode a membrane-bound form of casein kinase-1. Mol. Biol. Cell 3, 275-286.

Whyte, D. B., Kirschmeier,P., Hockenberry, T.N., Nunez-Oliva, I., James, L., Catino, J. J., Bishop, W. R., and Pai, J. K. (1997). $\mathrm{K}$ - and $\mathrm{N}$-Ras are geranylgeranylated in cells treated with farnesyl protein transferase inhibitors. J. Biol. Chem. 272, 14459-14464.

Wright, L. P., and Philips, M. R. (2006). Thematic review series: lipid posttranslational modifications. CAAX modification and membrane targeting of ras. J. Lipid Res. 47, 883-891.

Yamane, H.K., Farnsworth, C.C., Xie, H.Y., Howald, W., Fung, B. K., Clarke, S., Gelb, M. H., and Glomset, J. A. (1990). Brain $G$ protein gamma subunits contain an all-trans-geranylgeranylcysteine methyl ester at their carboxyl termini. Proc. Natl. Acad. Sci. U.S.A. 87, 5868-5872.

Conflict of Interest Statement: The authors declare that the research was conducted in the absence of any commercial or financial relationships that could be construed as a potential conflict of interest.

Received: 24 October 2010; paper pending published: 01 November 2010; accepted: 01 November 2010; published online: 23 November 2010.

Citation: Price CTD, Jones SC, Amundson KE and Kwaik YA (2010) Host-mediated post-translational prenylation of novelDot/ Icm-translocated effectors of Legionella pneumophila. Front. Microbio. 1:131. doi: 10.3389/fmicb.2010.00131

This article was submitted to Frontiers in Cellular and Infection Microbiology, a specialty of Frontiers in Microbiology.

Copyright () 2010 Price, Jones, Amundson and Kwaik. This is an open-access article subject to an exclusive license agreement between the authors and the Frontiers Research Foundation, which permits unrestricted use, distribution, and reproduction in any medium, provided the original authors and source are credited. 


\section{APPENDIX}

Table S1 | Primers used in this study.

\begin{tabular}{|c|c|}
\hline Primer name & Nucleotide sequence \\
\hline pelA cya F & GGATCCTTATGAGTGAATATTTGG \\
\hline pelA cya $R$ & CTGCAGCTACATGAGCACACAAACAG \\
\hline pelB cya $F$ & GGATCCTTATGTCAAATACTGTTTTAG \\
\hline pelB cya $R$ & CTGCAGTTATTTAATCAGGCAATC \\
\hline pelD cya F & GGATCCTTGTGTTCAAAAAAAAGC \\
\hline pelD cya $\mathrm{R}$ & CTGCAGTCACAATAAAGAACAATTA \\
\hline pelE cya F & GGATCCTTTTGCATCTTGAATTGC \\
\hline pelE cya R & CTGCAGTCATAACAAATTGCATGG \\
\hline pelF cya F & GGATCCTTATGCGTAGCAGAACAGAA \\
\hline pelF cya $\mathrm{R}$ & CTGCAGTTAGAGAATGCTGCATTGC \\
\hline pelH cya F & GGATCCTTGTGCTAATGGAATTCG \\
\hline pelH cya R & CTGCAGTTACATTATTGTACAACGG \\
\hline pell cya F & GGATCCTTATGAAATTTAAGATTGC \\
\hline pell cya $\mathrm{R}$ & CTGCAGTTACCATATGATGCAACGA \\
\hline $3 \times$ pelA F & GCGGCCGCATGAGTGAATATTTGG \\
\hline $3 \times$ pelA $R$ & TCTAGACTACATGAGCACACAAACAG \\
\hline $3 X$ pelB F & GCGGCCGCATGTCAAATACTGTTTTAG \\
\hline $3 X$ pelB $R$ & TCTAGATTATTTAATCAGGCAATC \\
\hline $3 X$ pelD F & GCGGCCGCATGTTCAAAAAAAAGC \\
\hline $3 \times$ pelD R & TCTAGATCACAATAAAGAACAATTA \\
\hline $3 X$ pelE F & GCGGCCGCATGCATCTTGAATTGC \\
\hline $3 X$ pelE $R$ & TCTAGATCATAACAAATTGCATGG \\
\hline $3 X$ pelF F & GCGGCCGCATGCGTAGCAGAACAGAA \\
\hline $3 X$ pelF $R$ & TCTAGATTAGAGAATGCTGCATTGC \\
\hline $3 X$ pelH F & GCGGCCGCATGCTAATGGAATTCG \\
\hline 3XpelH R & TCTAGATTACATTATTGTACAACGG \\
\hline $3 X$ pell $F$ & GCGGCCGCATGAAATTTAAGATTGC \\
\hline 3X pell R & TCTAGATTACCATATGATGCAACGA \\
\hline $3 X$ pelA CA F & /5Phos/GCTGTGCTCATGTAGTCTAGAGGATCCCGGG \\
\hline $3 \times$ pelA CA $R$ & /5Phos/AACAGATTCTTTCTCTGGTG \\
\hline $3 X$ pelB CA F & /5Phos/GCCCTGATTAAATAATCTAGAGGATCCCGGG \\
\hline $3 \times$ pelB CA R & /5Phos/ATCAATATAATTATTAATTCG \\
\hline $3 X$ pelD CA F & /5Phos/TGTTCTTTATTGTGATCTAGAGGATCCCGGG \\
\hline $3 \times$ pelD CA R & /5Phos/ATTATTTGAGCTGATAATACACC \\
\hline $3 X$ pelE CA F & /5Phos/TGCAATTTGTTATGATCTAGAGGATCCCGGG \\
\hline $3 X$ pelE CA R & /5Phos/TGGCGAGAATTTACTAATTTTC \\
\hline $3 X$ pelF CA F & /5Phos/TGCAGCATTCTCTAATCTAGAGGATCCCGGG \\
\hline $3 X$ pelF CA R & /5Phos/TTGCTCTGTGAACTTTGGTTTG \\
\hline $3 \times$ pelH CA F & /5Phos/TGTACAATAATGTAATCTAGAGGATCCCGGG \\
\hline $3 \times$ pelH CA R & /5Phos/ACGGTGTTTTTTAGTATCTTTG \\
\hline $3 \times$ pell CA F & /5Phos/TGCATCATATGGTAATCTAGAGGATCCCGGG \\
\hline 3X pell CA R & /5Phos/ACGATTCTTACTTATCATAGG \\
\hline
\end{tabular}

